\title{
Towards the Automation of Remedial Action Schemes Design
}

\author{
Hanyue Li, Komal Shetye, \\ Thomas Overbye, Katherine Davis \\ Department of Electrical and Computer Engineering \\ Texas A\&M University \\ College Station, TX, United States \\ $\{$ hanyueli, shetye, overbye, katedavis\}@tamu.edu
}

\author{
Shamina Hossain-McKenzie \\ R\&D Cybersecurity \\ Sandia National Laboratories \\ Albuquerque, NM, Unites States \\ shossai@sandia.gov
}

\begin{abstract}
The constantly evolving nature of the grid is compelling the design process of Remedial Action Schemes (RAS) to keep up with the changes. This paper proposes a flexible and computationally efficient approach to automatically determine RAS corrective actions that alleviate line overloading violations. Statistical and functional characteristics summarized from RAS implemented in real power systems are used to guide the design parameters. This paper also leverages sensitivity-based techniques to determine corrective actions for specific contingencies quickly without repeated numerical simulations.
\end{abstract}

\section{Introduction}

Remedial Action Schemes (RAS), also referred as Special Protection Systems (SPS), are operation schemes designed to detect anomalous or predefined system conditions, and take mitigation actions to maintain the reliability and stability of power systems. Such corrective actions may include adjusting status and values (MW and Mvar) of load and generation, and topology of the electric network [1]. RAS are often required when the system is at risk for thermal overloading, voltage instability, small signal instability, transient instability and cascading outages [2]. RAS are designed to take actions automatically when the triggering conditions are met, and resolve the issue within cycles to seconds for transient instability, and within minutes for thermal overloads [3].

In the past, RAS were implemented locally at substations or nearby control rooms. With the growing number of RAS being designed and applied in the system, and the expanding requirements to conduct wide-area operational studies more frequently, the power industry has largely embraced RAS as a centralized scheme in operation centers for the past decade [4]. The California Independent System Operator, for example, implemented RAS in its energy management system (EMS) in 2008, where RAS control actions are simulated in the real-time contingency analysis module [5]. Similarly, BC Hydro adopted RAS as a basic operation tool in the control room, where the arming of RAS is automatically executed by the transient stability assessment function [6]. Bonneville Power Administration also embedded the corrective schemes in its EMS. The RAS simulations are conducted for both current and future hour operational studies to maintain the system integrity [7].

The implementation of RAS in real-time operations provides comfort to the operators to help ensure the reliability and stability of the system after any credible contingencies and events. As the electric power industry is continually challenged with the increasing power transfer, new generation interconnections, aging equipment with limited capacity, and delays in constructing major transmission projects, RAS are a practical way of utilizing the existing infrastructure to maximize the power transfer capability reliably [8, 9].

Traditional RAS design currently takes a holistic approach that requires years of experience with the operations of a specific system [10]. New RAS are proposed to reliability coordinators individually by entities such as transmission owners, generator owners, and distribution providers based on their operational experience. Each RAS is modeled and introduced to the system individually to address some predetermined contingencies that are known to cause violations of reliability or stability standards [11]. To mitigate the potential violations or instabilities, numerous off-line simulations are repeated on those predefined scenarios to ensure that the candidate remedial action is sufficient, and does not introduce unintentional risks to the system. The implementation and testing of the RAS then involves manually defining the corrective action for a contingency definition within the EMS or other simulation software. The parameters associated with each scheme, such as the arming conditions, triggering threshold, and the numerical values of generation tripping and load shedding, usually do not change during 
real-time operations [12, 10].

Many research efforts, from both industry and academia, have gone into improving the flexibility and dynamics of RAS implementation. In [13], an event-based method was proposed to enhance RAS that are created to address specific frequency and voltage instability issues. Using transient energy analysis, the conventional RAS implementation can be adjusted with flexible triggering thresholds [14], and also adaptive corrective actions [15]. To mitigate the risk of voltage instability and voltage collapse, BC Hydro developed a methodology to determine the magnitude of load shedding based on real-time measurement data [16]. Recent work of [17] proposed an approach to adaptively set the arming parameters of existing RAS based on realistic and near real-time operation conditions. These RAS are automated in the sense that the parameters adapt to real-time conditions, however the design of the underlying RAS itself is manual.

In addition to the slow design process, the constantly evolving nature of the grid is compelling RAS to keep up with the changes. With the increasing penetration of distributed energy resources such as solar photovoltaic (PV) systems and wind farms, and grid-edge devices, and the rise of unpredictable disturbances, a traditional slow and manual RAS identification design process may not suffice. Also, to mitigate the impacts of these unpredictable events as they occur during grid operations, it is important to address the design with online time-frames in mind. The corrective actions need to be computed fast, within seconds or minutes, depending on the type of violation.

The power industry is cognizant of these evolving needs and hence there are regular assessments of the existing RAS' adequacy and the need for upgrades. For instance, in 2018 Hydro One in Canada replaced the RAS at their Bruce substation [18]. This increased the functionality to detect and operate for more contingencies and configurations, and enhanced system operation. Similarly, the need for and installation of new RAS are announced regularly by different entities [19. 20, 21, 22]. However, the design process still involves repeated, offline simulations.

Hence, this paper proposes a more flexible, computationally efficient approach to determine RAS corrective actions. The proposed RAS creation procedure aims to automatically generate triggering condition and corrective action pairs based on the identified need for new RAS creation. Using a 2000-bus synthetic system as an example, this paper focuses on RAS that are created for line overloading mitigation. Statistical and functional characteristics summarized from RAS implemented in real power systems are utilized as a reference to guide the design parameters. Building on prior work on online RAS [23] where line overloads were resolved by generation redispatch, this approach also leverages sensitivity-based techniques, and adds to it by considering two more types of actions, i.e. line switching and load shed and selects an "optimal" choice. Sensitivities such as line outage distribution factors (LODF) and transmission loading reliefs (TLR) are calculated rapidly using sparse vector methods [24] to design corrective actions for specific contingencies without repeated numerical simulations. Since this approach only uses electric grid topology and parameters as inputs for sensitivity analysis, it is general enough to be applied to different systems.

\section{Background}

\subsection{Traditional RAS Design Process}

The need to modify existing RAS or design a new RAS is usually identified during system planning studies, which can range from utility to interconnection scales [3]. From the utility scale, RAS entities can submit proposals to their reliability coordinator (RC) for RAS introduction, modification, and retirement. The RC is responsible for approving or providing revision comments after reviewing the proposal. On an interconnection scale, RCs are required to review the existing RAS within their territories every five years. All the deficiencies identified during this review process should be addressed by the corresponding RAS owners within six months [11].

According to the Western Electricity Coordination Council (WECC), there are four common elements for the design of RAS: arming criteria, initiating conditions, actions taken, and time requirements. The arming criteria are critical system conditions for which a step-wise RAS should be ready to take action when required. The initial conditions are the contingencies that have been know to cause violations of reliability and stability standards, which will initiate the RAS corrective action if the scheme is armed. The initial conditions can be event-based, parameter-based, response-based, or the combination of the above. Event-based schemes directly detect outages and/or fault events and initiate actions to fully or partially mitigate the event consequence. Parameter-based schemes measure variables for which a significant change confirms the occurrence of a critical event. Response-based schemes monitor system response during events and disturbances and incorporate a closed-loop process to react to actual system conditions [12]. The work of [10] finds that most RAS 
in the WECC system initiate upon changes to system topology, with very few being triggered by system condition changes.

For a critical contingency, various potential remedial actions are usually available to mitigate the violations and improve system performance. The RAS actions should at least result in an acceptable system operating condition after the occurrence of a critical contingency or a disturbance. The corrective action should not introduce unintentional risks or interfere with the performance of other RAS in the system. The corrective actions may include but are not limited to [3]:

- Islanding or other line opening

- Generator tripping

- Generator runback

- Load shedding

- Braking resistors

- Static VAR control units

- Capacitor and/or reactor switching

\subsection{Sensitivity Analysis and Sparse Vector Methods}

Sensitivities are linearized relationships, which help determine the impact of small changes in a variable on the system. TLR sensitivities gauge the sensitivity of a line flow to multiple power transfers, whereas LODF depicts how the outage of one more more lines, taken one at a time, affects the flows on the other lines. Both these sensitivities can be derived from the Injection Shift Factor (ISF) matrix represented by $\boldsymbol{\Psi}$ [25, 26, 27], which is constructed using the lossless dc power flow assumptions. The change in real power line flows is given as,

$$
\Delta \mathrm{f}=\Psi \Delta \mathrm{p}
$$

where $\Delta p$ is the vector of changes in the real power injection at each bus, and the element $\boldsymbol{\psi}_{l}^{n}$ in row $\boldsymbol{l}$ and column $\boldsymbol{n}$ of $\boldsymbol{\Psi}$ is the ISF of line $\boldsymbol{l}$ with respect to the injection at node $\boldsymbol{n}$. For a system with $\boldsymbol{L}$ lines and $\boldsymbol{N}$ nodes (buses), $\boldsymbol{\Psi}$ is defined as,

$$
\Psi=\tilde{\mathbf{B}} \mathbf{A}\left[\mathbf{B}^{\prime}\right]^{-1}
$$

where $\tilde{\mathbf{B}} \triangleq-\operatorname{diag}\left\{\boldsymbol{b}_{\mathbf{1}}, \boldsymbol{b}_{\mathbf{2}}, \ldots, \boldsymbol{b}_{\boldsymbol{L}}\right\} \quad\left(\boldsymbol{b}_{\boldsymbol{l}}\right.$ is the series susceptance of line $\boldsymbol{l}$ ), $\mathbf{A}$ is an $\mathbf{L} \times \mathbf{N}$ incidence matrix where the element $\boldsymbol{a}_{i j}$ is non-zero only when line $\boldsymbol{l}_{\boldsymbol{i}}$ is coincident with node $j$, and $\mathbf{B}^{\prime}=\mathbf{A}^{\mathbf{T}} \tilde{\mathbf{B}} \mathbf{A}$.

For the sensitivity calculations, all the elements of $\Psi$ are not needed as only certain lines and nodes are considered at a time, as will be shown below. Hence the full matrix as described in (2) does not need to be computed. Rather, the sensitivities of the elements of interest can be found using sparse vector methods [24]. For example, $\Delta \mathbf{p}$ is sparse in this case since the injection(s) contributed by the corrective action(s) are limited in number due to a judicious selection of the candidate nodes and the design criteria used. Because of this, fast forward substitution can be used. Similarly, monitoring a reasonable number of lines can help make $\boldsymbol{\Delta} \mathbf{f}$ sparse, and enable the use of fast backward substitution. This is able to substantially reduce the computational complexity. Once the matrix is factored (an $\mathbf{O}\left(\mathbf{n}^{\mathbf{1 . 4}}\right)$ ) operation, doing a fast forward and fast backward substitution involves very few (much less than $\mathbf{O}(\mathbf{n}))$ operations [28, 24].

\subsection{ACTIVSg2000 Synthetic System}

The ACTIVSg2000 synthetic system is a fictitious power network on the geographic footprint of Texas, United States. It includes detailed modeling of generators, loads, transmission lines, and other power system elements. This test case and its relating data are created using the methodology outlined in [29. 30] which captures the structural and functional characteristics of actual power grids. Figure 1 shows the one-line diagram of the ACTIVSg2000 synthetic transmission system. It has 2000 buses and 3206 transmission branches. The total electric load is 67 $\mathrm{GW}$ and the total generation capacity is $100 \mathrm{GW}$. The $500-\mathrm{kV}, 230-\mathrm{kV}$ and $115-\mathrm{kV}$ network in this test case are represented as the orange, purple and green lines in the one-line diagram, respectively. Similar to the actual grid in Texas, the wind generation resource is designed to be abundant in the far west and panhandle area. Load centers are in the east and central side of the system, where Dallas, Houston, and Austin metropolitan areas are located. Higher voltage transmission infrastructures are constructed to connect the generation facilities to the load clusters.

This test case has an initial ac power flow solution, and it is N-1 secure. It also contains several common control schemes and models such as switched shunts and load tap changers for reactive power control, generators that can remotely regulate voltages, phase shift transformers, automatic generation control (AGC), etc. On the dynamics side, it models the key generator controls such as the exciter, governor, and stabilizer, and even protection devices such as under-frequency load relays. Thus, while there are several controls in place to aid system security and stability in case of contingencies, there is no particular RAS developed for this test case. By developing RAS from scratch for a synthetic, realistic system, we propose a method to design new RAS in real grids. 


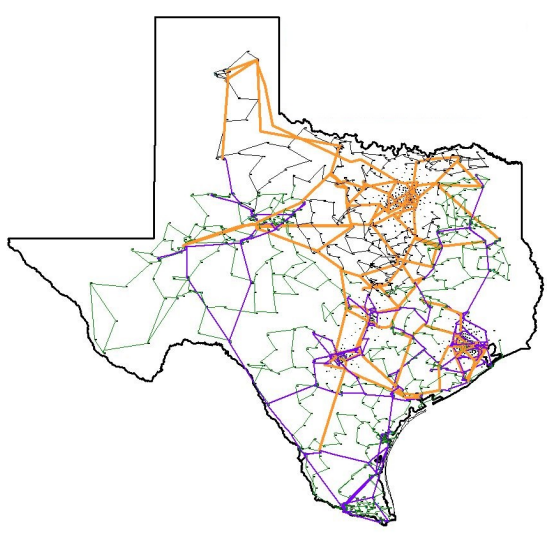

Figure 1. One-line diagram of the ACTIVSg2000 synthetic transmission model

\section{Automated RAS Design Procedure}

As mentioned earlier, the RAS design procedure in this paper automatically generates triggering condition and corrective action pairs, with a focus on line overloading mitigation. The design parameters are realistic as they are based on statistical and functional characteristics derived from actual RAS implementations. Sensitivity factors such as LODF and TLR are used to quickly design corrective actions for severe contingencies, eliminating the need for repeated numerical simulations to determine the corrective action(s). The 2000-bus synthetic system introduced above is used here as the test case.

\subsection{Problem Recognition}

Problem recognition is the process of detecting system operation violations, identifying the need for a RAS, and determining the characteristics required to mitigate the problems. In this paper, line overloading violations are determined using an intentionally constructed contingency list and transient stability studies solely for the purpose of providing input data to demonstrate the automatic RAS creation process.

3.1.1. Case Preparation: Transmission conductors are always designed to be operated under certain temperature limits to avoid damages to the equipment [31]. Transmission facilities usually determine multiple ratings for the conductors. Normal rating specifies the level of electrical loading that one transmission line can withstand through the daily demand cycles without loss of equipment life. Emergency rating defines the electrical loading that one transmission line can support for a finite period of time, while assuming acceptable loss of equipment life [31].

The violation of a transmission line normal thermal limit is usually not an urgent problem. In control rooms, established procedures exist to inform the operators on the actions needed to bring the transmission line flows down to acceptable thresholds [8]. Generator dispatch programs in real-time operation, such as security-constrained economic dispatch and security-constrained optimal power flow, aim to alleviate line violations by changing generator outputs as well [25]. However, in case of a severe event, transmission line loading may escalate quickly to violate reliability standards by exceeding its short-term emergency rating [32], or causing cascading tripping of lines [33]. Under such extreme scenarios where operators and embedded control programs do not have enough time to react, automatic corrective actions are needed to respond to the event immediately to prevent unacceptable system operating conditions.

Based on typical current ratings of conductors at each nominal voltage level, normal MVA limits of transmission lines are calculated and included during the creation process of the 2000-bus synthetic system [29]. In this paper, for different voltage levels, the ratio distribution of emergency MVA rating to normal MVA rating is summarized based on real transmission networks. This ratio distribution is then used to the estimate emergency rating of each transmission line in the 2000-bus synthetic network.

3.1.2. Triggering Condition Identification: To identify severe overloading conditions where RAS are needed in the synthetic system, a list of contingencies modeling the loss of two bulk electric system elements is created. This list considers two N-2 contingency categories defined in North American Electric Reliability Corporation (NERC) transmission planning performance requirements [34], where the multiple contingencies from a common structure (P7), and two overlapping single contingencies (P6) are considered.

As there are over 3,000 branches in the 2000-bus synthetic system, it is unrealistic to use a complete $\mathrm{N}-2$ contingency list that includes every possible combination. To effectively identify the set of contingencies with severe consequences, the list is reduced to only consider double line outage of branches $161 \mathrm{kV}$ and above for P7 events. For P6 events, an LODF screening tool is used to identify pairs of contingencies that are significant without solving all the contingencies [35]. Only those contingency pairs that create line limit monitoring violations are included in the contingency list. 
These contingencies are then modeled in a transient stability simulation. The simulation duration is set to be 20 seconds, with a half-cycle time step. The MVA flows of transmission lines $115 \mathrm{kV}$ and above are monitored for emergency rating violations. If there is at least one branch with a sustained line overloading issue after the outage or fault is applied, this contingency is considered to be severe and in need of a RAS.

\subsection{Corrective Actions}

3.2.1. Industrial RAS Statistics: To guide the design of corrective action for each line overloading contingency identified in the previous steps, statistical analysis was conducted on 41 RAS implemented in real power systems to summarize their key features and range of parameter values. All of the industrial RAS used here are designed to alleviate one or multiple transmission line thermal violations. Figure 2 shows the four categories of corrective actions adopted by these industrial RAS. Generator adjusting, which includes the opening of generator units and changing generator output set points, is the most commonly used action to mitigate line overloads. Branch switching, and load shedding are two other popular schemes adopted by the industry. Some thermal violations also require hybrid actions with a mix of generator adjusting, branch opening and load shedding.

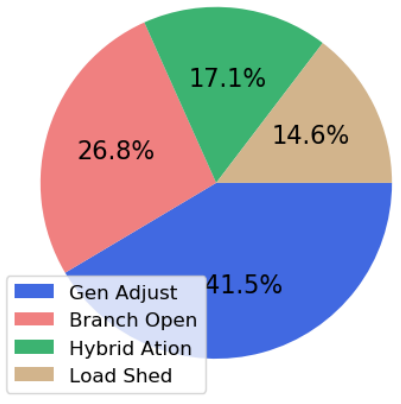

Figure 2. Corrective Action Types for Overload Mitigation

Table 1 describes the characteristics and range of key parameters for each corrective action type. For branch switching schemes, the total number and MVA capcity of lines opened, the number of nodes away from the overloading line, and their nominal voltage levels are recorded. For generator adjusting, the number and MW value of generators adjusted, their distance from the overloading line, and their fuel types are used as reference for the corrective action design. For load shedding, the number and MW value of load opened, and the number of nodes away from the overloading line are also noted.

\begin{tabular}{|l|l|l|l|}
\hline $\begin{array}{l}\text { Element } \\
\text { Type }\end{array}$ & \multicolumn{1}{|c|}{ Characteristics } & Range & Avg \\
\hline \multirow{5}{*}{ Branch } & $\begin{array}{l}\text { Number of branch } \\
\text { switched }\end{array}$ & $1-2$ & 1 \\
\cline { 2 - 4 } & Capacity of branch (MVA) & $\begin{array}{l}30- \\
500\end{array}$ & 223 \\
\cline { 2 - 4 } & $\begin{array}{l}\text { Distance from overloading } \\
\text { line (nodes) }\end{array}$ & $1-3$ & 2 \\
\cline { 2 - 4 } & Nominal voltage (kV) & $69-765$ & 230 \\
\hline \multirow{5}{*}{ Generator } & Number of gen adjusted & $1-10$ & 3 \\
\cline { 2 - 4 } & Size of gen adjusted (MW) & $\begin{array}{l}42- \\
350\end{array}$ & 120 \\
\cline { 2 - 4 } & $\begin{array}{l}\text { Distance from overloading } \\
\text { line (nodes) }\end{array}$ & $1-12$ & 4 \\
\cline { 2 - 4 } & Fuel type & $\begin{array}{l}\text { wind, } \\
\text { solar, } \\
\text { NG }\end{array}$ & - \\
\hline \multirow{5}{*}{ Load } & Number of load opened & $1-8$ & 5 \\
\cline { 2 - 4 } & $\begin{array}{l}\text { Size of load adjusted } \\
\text { (MW) }\end{array}$ & $12-75$ & 21 \\
\cline { 2 - 4 } & $\begin{array}{l}\text { Distance from overloading } \\
\text { line (nodes) }\end{array}$ & $1-10$ & 6 \\
\hline
\end{tabular}

Table 1. Industrial RAS Corrective Action Statistics

3.2.2. Corrective Action Design: The procedure of creating automated corrective actions is illustrated in Figure 3 Information of the overloading lines identified in the problem recognition step is used as the input to shortlist the controllable elements that can be used for the remedial actions. For a specific overloading line, referencing Table 1, branches above $69-\mathrm{kV}$ and also within three nodes away are considered to be controllable equipment. Besides the distance from thermal violation location, generators are filtered out based on their fuel types, where only wind, solar and natural gas units will be used to develop corrective actions. Loads that can be reduced to mitigate the overloads are selected only based on their locations relative to the overloading lines. This step limits the list of controllable elements to a small number, so that the real power injection change vector $\Delta \mathrm{p}$ mentioned in the background section is not full and sparse vector methods can be applied to reduce the computation time.

To ensure that corrective actions can be automatically created within the online simulation time frame, sensitivity-based techniques are leveraged to study the relationships between the controllable elements and the overloading lines. To design the 
candidate branch switching scheme, the LODF matrix represented by $\mathbf{d}$ is derived from the ISF matrix $\Psi$. An element $\boldsymbol{d}_{l}^{k}$ of this matrix $\mathbf{d}$ can be written as,

$$
d_{l}^{k}=\frac{\psi_{l}^{m}-\psi_{l}^{n}}{1-\left(\psi_{k}^{m}-\psi_{k}^{n}\right)}
$$

where $\boldsymbol{m}$ and $\boldsymbol{n}$ are the two nodes associated with the switched line $\boldsymbol{k}$.

The real power flow change on line $l$ due to the switching of line $\boldsymbol{k}$ is given as,

$$
\Delta f_{l}=d f_{k}
$$

where $f_{k}$ is the pre-switching real power flow on line $\boldsymbol{k}$. The branches selected to be controllable elements are sorted by their pre-outaged flows, and LODF sensitivity to the overloading lines. For the branch opening scheme, those branches are switched open in this order until the thermal violations are estimated to be alleviated.

The generator adjusting and load shedding candidate actions are determined using TLR sensitivities. The TLR sensitivity matrix is also derived from $\Psi$. It estimates the impact of multiple transfers on one transmission line. An element $\phi_{l}^{w}$ of this TLR sensitivity matrix $\Phi$ can be written as,

$$
\phi_{l}^{w}=\psi_{l}^{m}-\psi_{l}^{n}
$$

where $l$ is the transmission line of interest, and two nodes $\boldsymbol{m}$ and $\boldsymbol{n}$ define one transaction $\boldsymbol{w}$.

The real power flow change on line $l$ due to the transaction $\boldsymbol{w}$ is given as,

$$
\Delta f_{l}=\phi_{l}^{w} \Delta t
$$

where $\Delta t$ is the amount of MW transfer associated with transaction $\boldsymbol{w}$. In this work, the adjustments made on generation and load values and status are modeled as transactions. Similar to the branch switching scheme, the generators and loads selected to be controllable elements are sorted based on their TLR sensitivities and current generation or load MW values. This determines the order of operation until the overloading issue is mitigated.

The three candidate actions are then evaluated based on their effectiveness and simplicity. The final proposed RAS is selected based on this evaluation. The effectiveness of a RAS means that the corrective action can successfully mitigate the overloading issues as intended without introducing any unexpected violations. This is quantified by the post-contingency loading on the targeted lines that have exhibited violations without the implementation of RAS. The simplicity of the candidate action is defined as the total number of elements involved in one scheme.
This methodology is illustrated in the next section with two examples, however, its description so far has shown that it is general enough to be applied to most systems, to design RAS for line overloads.

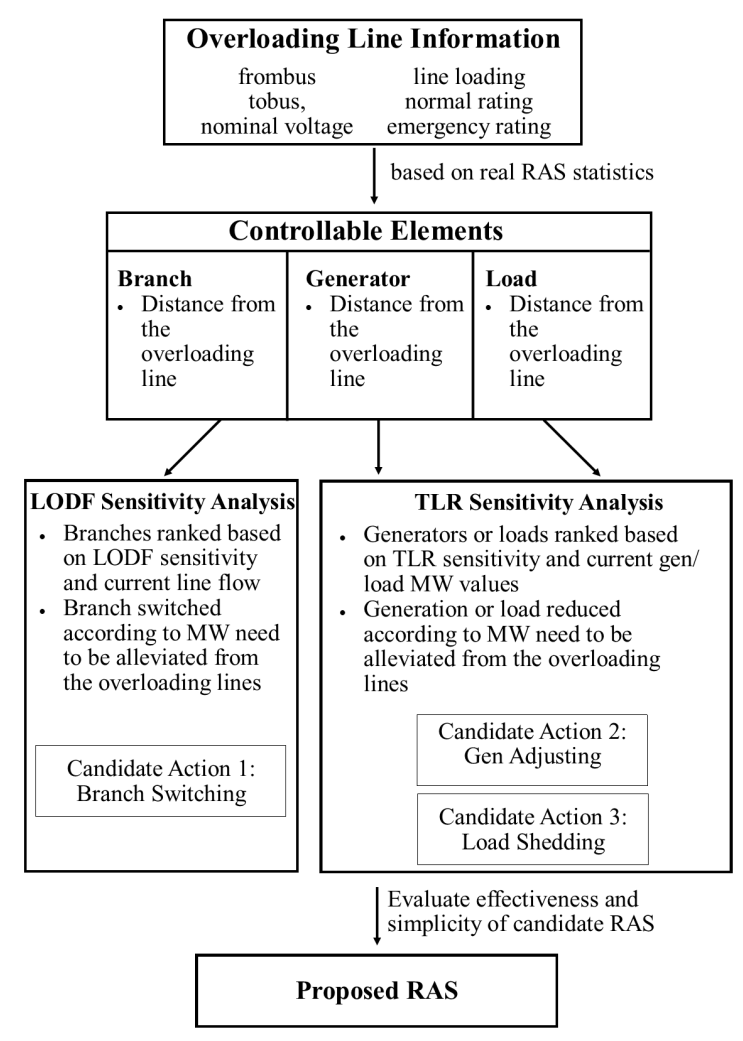

Figure 3. Automated RAS Procedure Flow Chart

\section{ACTIVSg2000 Example RAS}

The proposed automated RAS procedure shown in Figure 3 is illustrated for application in two examples in this section. The examples consider the risk of thermal overload on transmission lines and transformers. The real world is concerned with additional risks, including voltage instability, small signal instability, transient instability and cascading outages. The options for corrective actions are load shedding, line switching, and generator output adjustments.

\subsection{Example RAS 1}

The first example RAS created is located in West Texas, an area with concentrated wind resources. Substations constructed to house wind generation facilities are interconnected with a $115 \mathrm{kV}$ network in the synthetic system. Figure 4 is a one-line diagram showing the pre-contingency system conditions of this 
region. The percentage line flows are shown using pie charts [36]. The transmission system is loaded around 30 to 50 percent when all the wind units are producing electricity, and all the $115 \mathrm{kV}$ lines are online.

However, a severe contingency is identified in the region. Illustrated in Figure 5, with the loss of two $115 \mathrm{kV}$ transmission lines, NOLAN 0 to TUSCOLA 0 , and NOLAN 0 to TUSOCOLA 1 , the branch flow between substation NOLAN and TRENT would surge to $110 \%$ of its emergency rating, which is considered as an unacceptable operating condition.

To mitigate the impact of this contingency, a list of generators, loads and branches are chosen as controllable devices for corrective actions. Within this element list, the wind generator at substation NOLAN is directly connected to the bus related to the overloading line. As the other two lines coming out of NOLAN substation are outaged, $100 \%$ of the generation reduction from this generator is reflected as the decrease of flow from NOLAN 0 to TRENT 01 . Due to its simplicity and effectiveness, the opening of this generator is chosen to be the corrective action. Shown in Figure 6 if the severe contingency is detected, the $127 \mathrm{MW}$ wind generator at NOLAN substation would open, and relieve the NOLAN 0 to TRENT 01 line from $110 \%$ loading to $1 \%$ loading. On an Intel ${ }^{\circledR} \mathrm{Xeon}^{\circledR}$ E5-1650 V4 CPU @ 3.6GHz with 32GB RAM, 64-bit OS, $\mathrm{x}-64$ based processor, the corrective actions can be created within a few seconds.

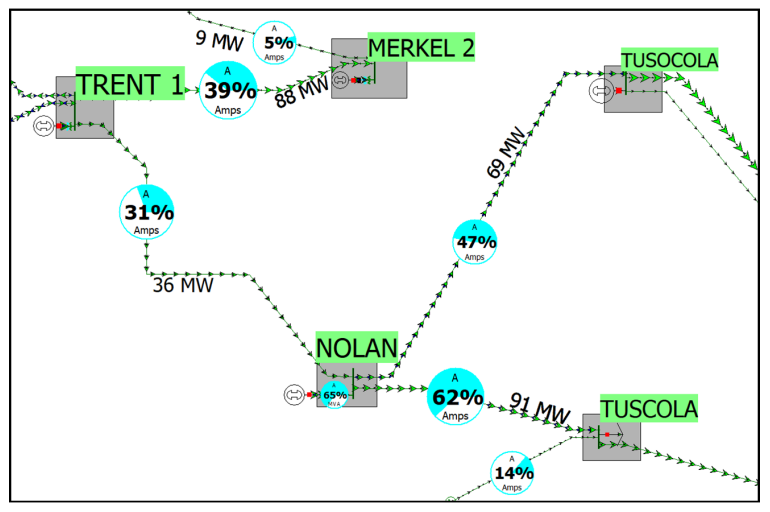

Figure 4. ACTIVSg2000 Example 1: Pre-Contingency

\subsection{Example RAS 2}

The second example RAS is located in the Dallas metropolitan area, where load clusters are connected with a $161 \mathrm{kV}$ network in the synthetic system. Figure 7 is a one-line diagram showing the pre-contingency system conditions of this area. During the summer peak scenario that the synthetic case represents, the most

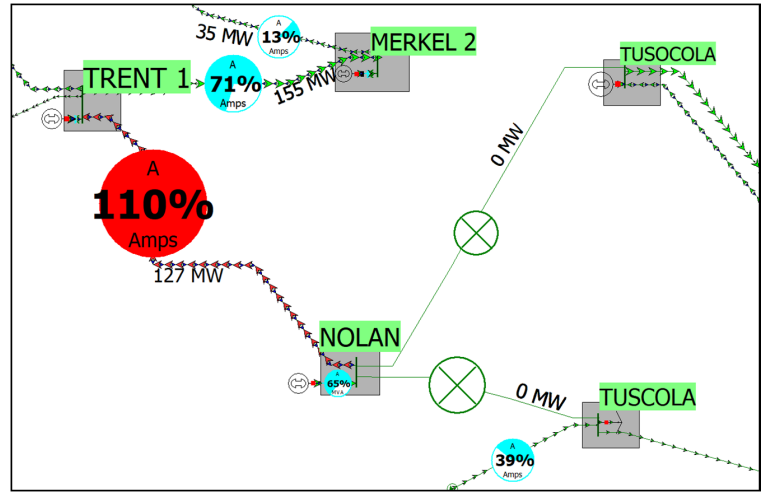

Figure 5. ACTIVSg2000 Example 1: Post-Contingency, without RAS

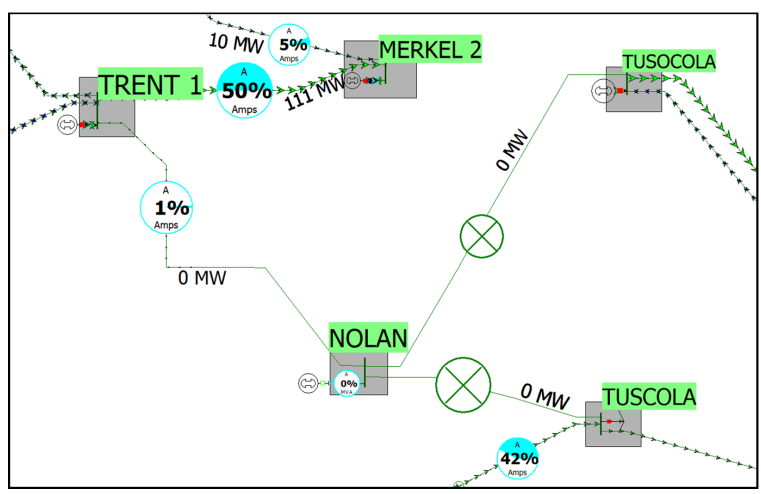

Figure 6. ACTIVSg2000 Example 1: Post-Contingency, with RAS

loaded line in the system is DALLAS 31 to DALLAS 36.

Illustrated in Figure 8, after the loss of two 161 $\mathrm{kV}$ transmission lines, DALLAS 21 to DALLAS 23, and DALLAS 25 to DALLAS 23, the branch flow between substation DALLAS 31 and DALLAS 36 would increase to $103 \%$ of its emergency rating without the implementation of a RAS.

Based on the bus numbers related to this line violation, a list of loads and branches are chosen as controllable devices for corrective actions. Here, the option to use generator adjusting as a corrective action is eliminated since there is no generator nearby. Two candidate corrective actions that focus on load shedding and branch switching respectively are evaluated. In the first candidate action, the $67 \mathrm{MW}$ load at substation DALLAS 23 is proposed to be taken offline. This load is 1 node away from the the overloading line. According to the TLR analysis, $57.6 \%$ of the load reduction from this location is reflected as the decrease of flow on the overloading line. In the second candidate action, the transmission line between ADDISON and DALLAS 31 
is switched open. This branch is loaded at 89 MVA before the contingency, and the LODF analysis shows that a 45 MVA reduction can be seen on the overloading branch from the line switching. As the branch switching scheme has a larger alleviation on the overloading line, and does not require service interruption to customers, it is selected to be the corrective action. This corrective action also only takes a few seconds to generate using the computer system described in example RAS 1.

Figures 9 and 10 also compare the effectiveness of the two candidate actions. With load shedding, the overloading line is reduced to $87 \%$, while with branch switching, the loading is reduced to $62 \%$.

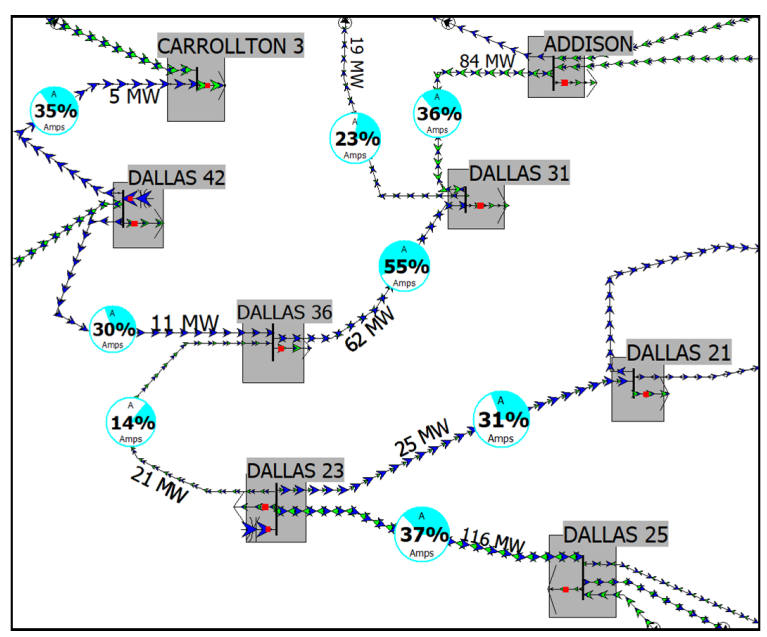

Figure 7. ACTIVSg2000 Example 2: Pre-Contingency

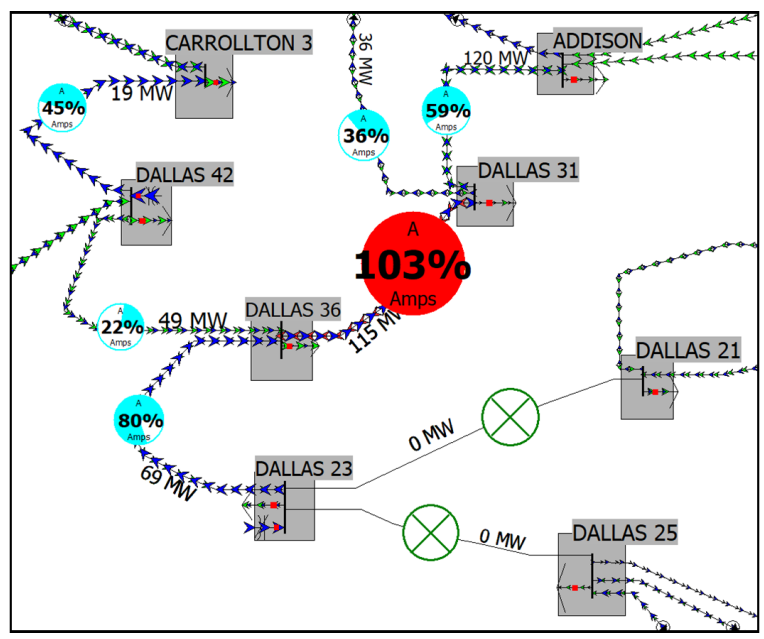

Figure 8. ACTIVSg2000 Example 2: Post-Contingency, without RAS

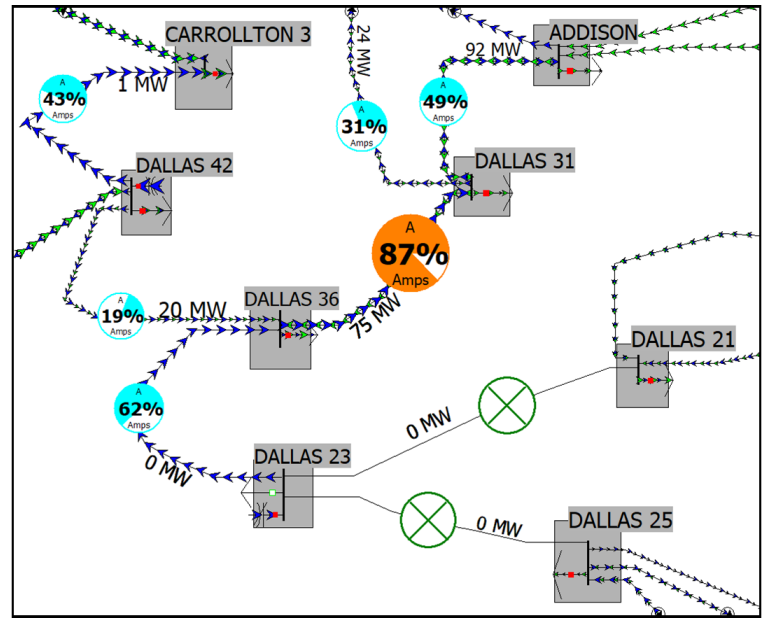

Figure 9. ACTIVSg2000 Example 2:

Post-Contingency, with Candidate Action 1

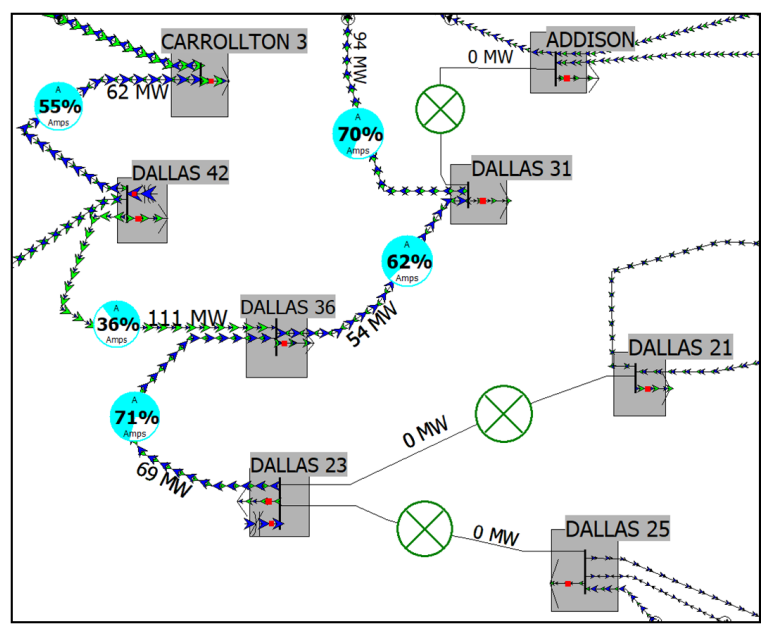

Figure 10. ACTIVSg2000 Example 2:

Post-Contingency, with Candidate Action 2

\section{Conclusions and Future Work}

The constantly evolving nature of the grid is compelling the design process of RAS to keep up with the changes. To address this issue, this paper has proposed a flexible and computationally efficient approach to determine RAS corrective actions that alleviate line overloading violations. Statistical and functional characteristics summarized from RAS implemented in real power systems are used to guide the design parameters. This paper also leverages sensitivity-based techniques such as LODF and TLR to determine corrective actions for specific contingencies rapidly without repeated numerical simulations. This proposed method provides a useful methodology for power system real-time operations, as the corrective 
actions are determined only within seconds for a 2000-bus realistic test system. Following this approach, it is expected that based on 1) the size/complexity of the system and 2) the type and severity of the violations, the unpredictable events could be addressed in a timely (i.e. seconds to minutes) and reliable manner.

This work sets the path forward towards a fully automated remedial action scheme design process. Future development includes, but is not limited to the following items.

- Developing an online screening tool for problem recognition that detects system operating violations and identifies the need for a RAS rapidly.

- Expanding the scope to address other operation problems such as voltage violations and instabilities.

- Utilizing stochastic scenarios in the synthetic system to dynamically calculate RAS parameters such as time delays or load shedding amounts.

- Developing more comprehensive RAS evaluation metrics that quantifies system performance and complexity of proposed RAS.

\section{Acknowledgement}

This work was supported in part by the U.S. Department of Energy Advanced Research Projects Agency Energy (ARPA-E), and in part by the Laboratory Directed Research \& Development (LDRD) at Sandia National Laboratories.

\section{References}

[1] North American Electric Reliability Corporation (NERC), "Glossary of Terms Used in NERC Reliability Standards." https://www.nerc.com/files/glossary_of_terms.pdf 2020.

[2] Y. Zhang, M. E. Raoufat, and K. Tomsovic, "Remedial action schemes and defense systems," Smart Grid Handbook, pp. 1-10, 2016

[3] Western Electricity Coordinating Council (WECC), "Remedial Action Scheme Design Guide," 2016.

[4] J. McCalley, O. Oluwaseyi, V. Krishnan, R. Dai, C. Singh, and K. Jiang, "System protection schemes: limitations, risks, and management," Final Report to the Power Systems Engineering Research Center (PSERC), 2010.

[5] M. Varghese, L. Jin, S. Ghosh, G. Lin, and B. Pek, "The CAISO experience of implementing automated remedial action schemes in energy management systems," in 2009 IEEE Power \& Energy Society General Meeting, pp. 1-5, IEEE, 2009.

[6] S. C. Pai and J. Sun, "BCTC's experience towards a smarter grid-increasing limits and reliability with centralized intelligence remedial action schemes," in
2008 IEEE Canada Electric Power Conference, pp. 1-7, IEEE, 2008.

[7] R. Ramanathan, B. Tuck, and J. O'Brien, "BPA's experience of implementing remedial action schemes in power flow for operation studies," in 2013 IEEE Power \& Energy Society General Meeting, pp. 1-5, IEEE, 2013.

[8] E. Vaahedi, Practical power system operation. John Wiley \& Sons, 2014.

[9] J. Wen, P. Arons, and W. E. Liu, "The role of remedial action schemes in renewable generation integrations," in 2010 Innovative Smart Grid Technologies (ISGT), pp. 1-6, IEEE, 2010.

[10] J. G. O'Brien, E. L. Barrett, X. Fan, R. Diao, R. Huang, and Q. Huang, "Survey on RAS/SPS modeling practice," tech. rep., Pacific Northwest National Lab.(PNNL), Richland, WA (United States), 2017.

[11] North American Electric Reliability Corporation (NERC), "PRC-012-2 Remedial Action Schemes," 2016.

[12] P. Anderson and B. LeReverend, "Industry experience with special protection schemes," IEEE Transactions on Power Systems, vol. 11, no. 3, pp. 1166-1179, 1996.

[13] M. D. Maram and N. Amjady, "Event-based remedial action scheme against super-component contingencies to avert frequency and voltage instabilities," IET Generation, Transmission \& Distribution, vol. 8, no. 9, pp. 1591-1603, 2014.

[14] S. Wang and G. Rodriguez, "Smart RAS (Remedial Action Scheme)," in 2010 Innovative Smart Grid Technologies (ISGT), pp. 1-6, IEEE, 2010.

[15] Y. Zhang and K. Tomsovic, "Adaptive remedial action scheme based on transient energy analysis," in IEEE PES Power Systems Conference and Exposition, 2004., pp. 925-931, IEEE, 2004.

[16] H. Atighechi, P. Hu, J. Lu, G. Wang, and S. Ebrahimi, "A fast load shedding remedial action scheme using real-time data for bc hydro system," in 2016 IEEE Power and Energy Society General Meeting (PESGM), pp. 1-5, IEEE, 2016.

[17] X. Fan, R. Huang, Q. Huang, X. Li, E. L. Barrett, J. G. O'Brien, Z. Hou, H. Ren, S. Kincic, and H. Zhang, "Adaptive ras/sps system setting for improving grid reliability and asset utilization through predictive simulation and controls: A use case for transformative remedial action scheme tool (trast): Jim bridger ras evaluation and analysis," tech. rep., Pacific Northwest National Lab.(PNNL), Richland, WA (United States), 2019.

[18] "Reliability Outlook: An adequacy assessment of Ontario's electricity system (From January 2019 to December 2023)," tech. rep., Independent Electricity System Operator, Ontario (Canada), 2018.

[19] California ISO, “2016-2017 ISO Transmission Plan." https://www.caiso.com/Documents/AppendixF_Draft_ 2016-2017TransmissionPlan.pdt 2016.

[20] Dominion Virginia Power, "PJM New Remedial Action Scheme." https://www. pjm.com/ /media/committees-groups/committees/oc/ 20170110/20170110-item-20-temporary-dominion-ras. ashx 2017.

[21] ERCOT, "Proposal to Add Rocksprings Remedial Action Scheme." http://www.ercot.com/services/comm/ mkt_notices/archives/4386 2020. 
[22] ERCOT, "Proposal to Modify Wirtz Remedial Action Scheme." http://www.ercot.com/services/comm/ mkt_notices/archives/4493, 2020.

[23] S. Hossain-McKenzie, M. Kazerooni, K. Davis, S. Etigowni, and S. Zonouz, "Analytic corrective control selection for online remedial action scheme design in a cyber adversarial environment," IET Cyber-Physical Systems: Theory Applications, vol. 2, no. 4, pp. 188-197, 2017.

[24] W. F. Tinney, V. Brandwajn, and S. M. Chan, "Sparse vector methods," IEEE Transactions on Power Apparatus and Systems, vol. PAS-104, no. 2, pp. 295-301, 1985.

[25] A. J. Wood, B. F. Wollenberg, and G. B. Sheblé, Power generation, operation, and control. John Wiley \& Sons, 2013.

[26] M. Liu and G. Gross, "Effectiveness of the distribution factor approximations used in congestion modeling," in Proceedings of the 14th Power Systems Computation Conference, pp. 1-8, 2002.

[27] T. Guler, G. Gross, and M. Liu, "Generalized line outage distribution factors," IEEE Transactions on Power Systems, vol. 22, no. 2, pp. 879-881, 2007.

[28] F. L. Alvarado, "Computational complexity in power systems," IEEE Transactions on Power Apparatus and Systems, vol. 95, no. 4, pp. 1028-1037, 1976.

[29] A. B. Birchfield, T. Xu, K. M. Gegner, K. S. Shetye, and T. J. Overbye, "Grid structural characteristics as validation criteria for synthetic networks," IEEE Transactions on Power Systems, vol. 32, pp. 3258-3265, July 2017.

[30] H. Li, J. H. Yeo, A. Bornsheuer, and T. J. Overbye, “The creation and validation of load time series for synthetic electric power systems," IEEE Transactions on Power Systems, pp. 1-1, 2020.

[31] OATI OASIS, "Transmission Facilities Rating Methodology," 2013.

[32] North American Electric Reliability Corporation (NERC), "System Operating Limit Definition and Exceedance Clarification," 2016.

[33] W. Lachs, "Transmission-line overloads: real-time control," in IEE Proceedings C (Generation, Transmission and Distribution), vol. 134, pp. 342-347, IET, 1987.

[34] North American Electric Reliability Corporation (NERC), “TPL-001-4," 2013.

[35] C. M. Davis and T. J. Overbye, "Multiple element contingency screening," IEEE Transactions on Power Systems, vol. 26, no. 3, pp. 1294-1301, 2010.

[36] T. J. Overbye, J. D. Weber, and K. J. Pattern, "Analysis and visualization of market power in electric power systems," in Proceedings of the 32nd Annual Hawaii International Conference on Systems Sciences, Maui, HI, vol. Track3, January 1999. 